Received: 2017.01.21

Accepted: 2017.04 .05
Published: 2017.12.15

\section{Kaleidoscopic View of Bowel Tuberculosis on Multi- Detector Computed Tomography (CT) Enterography - A Novel Technique Unfolding an Archaic Disease}

Authors' Contribution:
A Study Design
B Data Collection
C Statistical Analysis
D Data Interpretation
E Manuscript Preparation
F Literature Search
G Funds Collection

\author{
Shuchi Bhatt ${ }^{\text {1ABCDEF }}$, Satarupa Roy ${ }^{1 \text { BDE }}$, Naveen Bhardwaj ${ }^{2 \mathrm{ABC}}$, Anupama Tandon ${ }^{1 \mathrm{BCDF}}$,

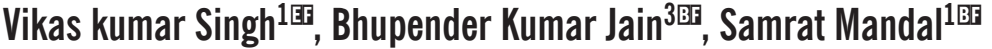 \\ ${ }^{1}$ Department of Radio-diagnosis, University College of Medical Sciences (University of Delhi) and Guru Teg Bahadur (GTB) \\ Hospital, Delhi, India \\ 2 Department of Radiology, SGT Medical College, Hospital and Research Institute, Gurgaon, India \\ ${ }^{3}$ Department of of Surgery, University College of Medical Sciences (University of Delhi) and Guru Teg Bahadur (GTB) Hospital, \\ Delhi, India
}

Author's address: Shuchi Bhatt, Deptartment of Radio-diagnosis, University College of Medical Sciences (University of Delhi) and Guru Teg Bahadur (GTB) Hospital, Dilshad Garden, Delhi-95, India, e-mail: drshuchi@hotmail.com

\author{
MeSH Keywords: $\quad$ Ileocecal Valve • Multidetector Computed Tomography • Tuberculosis \\ PDF file: $\quad$ http://www.polradiol.com/abstract/index/idArt/903473
}

Gastrointestinal tuberculosis (GI TB) is an important manifestation of abdominal tuberculosis (TB), an extra-pulmonary form of the disease. GI TB commonly affects the small bowel but is difficult to diagnose due to the challenge of evaluating the entire length of overlapping small bowel loops with traditional diagnostic methods like Barium meal follow through, abdominal computed tomography (CT), and endoscopy. New techniques of CT/MR enteroclysis/enterography are now available which specifically image the small bowel.

MDCT enterography (MDCTE) is a non-invasive, simple to perform, modified abdominal CT imaging technique permitting reasonably accurate evaluation of the small bowel lumen, wall, perienteric tissues, and solid organs within the abdomen. As GI TB can cause morphological alterations in and around the small bowel, MDCTE seems to be an attractive modality for patients suspected of abdominal or GI TB.

As scarce literature is available on MDCTE on tuberculosis, we present a pictorial review on MDCTE findings in patients with GI tuberculosis proved on FNAC and clinical and/or imaging follow-up.

\section{Background}

Extrapulmonary tuberculosis (TB) accounts for a significant number of tuberculosis cases in the developing world and is also increasingly encountered in the western world due to the rising incidence of human immune deficiency virus, use of immunosuppressant drugs, and immigration. Abdominal TB is the sixth most common manifestation of TB, and it may involve the lymph nodes, genitourinary tract, peritoneum or the gastrointestinal (GI) tract in a decreasing order [1]. GI TB may occur in isolation or as part of disseminated disease; with affection of the terminal ileum and ileocaecal (IC) junction in most cases [2]. It closely resembles Crohn's disease, in terms of clinical presentation and imaging and both diseases pose a great diagnostic difficulty to clinicians, radiologists, and endoscopists. Differentiation among the two inflammatory small bowel pathologies is essential as the basic treatment differs and institution of anti-tubercular treatment is required in GI TB. Comprehensive transverse and longitudinal evaluation of the entire small bowel is required for the diagnosis, which is not possible with a single imaging modality like barium meal follow through, enteroclysis, or abdominal computed tomography (CT). Imaging techniques targeted at 
the entire length of the superimposed bowel loops and the surrounding tissues are required for specific imaging of the small bowel in suspected cases.

A modified but noninvasive abdominopelvic CT technique called CT enterography was developed by Raptopoulos et al. in 1997 [3] to evaluate the extent and severity of Crohn's disease, especially in the small bowel. [3,4]. Administration of large volumes of oral contrast achieves optimal small bowel distention, thereby allowing superior visualisation and better assessment of the small bowel besides the usual evaluation of the surrounding tissues [5]. Further, use of MDCT for CT enterography enhances the spatial resolution in addition to a good small bowel distention. Bowel distension is achieved by ingestion of large volumes of oral contrast, unlike in CT enteroclysis whereby oral contrast is given through a tube placed at the duodenojejunal junction. This obviates the need for fluoroscopic nasoduodenal intubation, making CT enterography a better tolerated technique that also involves less radiation, time, and cost [4]. Moreover, it does not require an expertise to distend the bowel for imaging unlike in CT enteroclysis. 1200 to 1800 $\mathrm{ml}$ of positive or neutral oral contrast is given orally in a graded fashion to distend the bowel before the acquisition. An abnormally increased enhancement of the intestinal mucosa and the bowel wall in the regions of involvement is clearly demonstrated against the background of neutral luminal contrast [6]. Positive contrast should be used if complex peritoneal collections are present with a clinical suspicion of bowel perforation or presence of bowel fistulas in neglected cases of bowel tuberculosis. Positive contrast helps in good delineation of fistulas and the exact site of anatomical small bowel obstruction [7].

MDCTE comprehensively evaluates the small bowel lumen, mucosa, and wall along with the perienteric tissues, mesentery, peritoneum, and solid organs, making it a useful investigation in detecting small bowel disease $[4,6]$. It overcomes the limitations of both conventional 'barium meal follow through' in evaluating low-grade obstructions and extra-luminal disease and that of abdominal CT by achieving a good bowel distension and isovolumetric imaging.

\section{Technique}

Thin axial sections are obtained after an intake of 1500-1800 $\mathrm{ml}$ of oral contrast (positive or negative), extending from the diaphragm to the pubic symphysis. The protocol parameters are as follows: $\mathrm{kV}, 120$; effective $\mathrm{mAs}, 165$; detector collimation, $0.625 \times 64$. $80-120 \mathrm{ml}$ of non-ionic $(300 \mathrm{mg} / \mathrm{ml})$ contrast at a rate of $3 \mathrm{ml} / \mathrm{sec}$ is administered intravenously. The contrast scanning is done during the enteric $(45-50 \mathrm{~s})$ phase of contrast enhancement to acquire maximum information on the bowel. The source axial images are transferred to the workstation for formation of MPRs and volumetric images for better assessment of the morphological alterations and better depiction of the pathoanatomy of the bowel.

A single examination of CT enterography delivers around 12-20 mSv [8] of effective radiation dose to the patient. As multiple studies result in cumulative radiation exposure, its judicious use with efforts directed to reduce the radiation dose are advised [9].

\section{MDCT Enterography Findings}

\section{Normal}

An adequate study shows well-distended small bowel loops (duodenum, jejunum, ileum) with enhancement of the bowel wall when negative oral contrast is used (Figure 1).

Normal ileal and jejunal loops measure below $2.5 \mathrm{~cm}$ and $3 \mathrm{~cm}$ in calibre, respectively [7] with wall thickness up to $3 \mathrm{~mm}$ in the distended and $5 \mathrm{~mm}$ in the collapsed state and uniform wall enhancement [7]. A normal but collapsed bowel loop may appear slightly more enhancing than a distended loop. There should be no clumping together with abnormal segmental dilation/prominence of bowel loops. The caecum is positioned in the right iliac fossa, appears well distended with rounded configuration, showing normal walls, normal wall thickness and enhancement. The normal ileocaecal (IC) angle is 90 degrees and does not show any narrowing, widening, thickening, or abnormal wall enhancement (Figure 2).

Sometimes, differentiation of an abnormal bowel segment from a collapsed bowel segment is difficult but important to detect or rule out the pathology. Comparison to the adjacent normally distended segment is essential for a correct interpretation.

\section{GI tuberculosis}

GI TB may occur as an isolated disease or as part of a disseminated disease process. GI involvement occurs due to direct affection of the small bowel or due to nodal or peritoneal involvement. GI TB has a varied clinical presentation mandating early diagnosis.

Terminal ileum and IC junction are the most common involved sites followed by ileal loops, caecum, jejunal loops, and the remaining part of the colon. The rectum, duodenum, and stomach are rarely involved. Morphologically, bowel TB may present as a hypertrophic, ulcero-proliferative, and ulcerative form in 10\%,30\%, and $60 \%$, respectively [10]. Post-treatment healing with fibrosis results in formation of fibrotic strictures which may cause bowel obstruction.

\section{Level of bowel involvement}

The ileocaecal (IC) junction is involved in $64 \%$ of patients with GI tuberculosis [11]. Concurrent involvement of the caecum with the IC junction is suggestive of tuberculosis; a simultaneous ileal involvement is strongly suggestive of tuberculosis [12].

The IC junction displays abnormal mural thickening, abnormal position, and angulation. Thickening can be symmetric or asymmetric but concentric, with or without the presence of a stricture (decreased calibre with proximal dilatation), abnormal mural enhancement, and an altered IC angle (Figure 3, 4).

A concentric mural thickening of variable degrees (3 $\mathrm{mm}$ to $10 \mathrm{~mm}$ or more), usually showing homogenous 


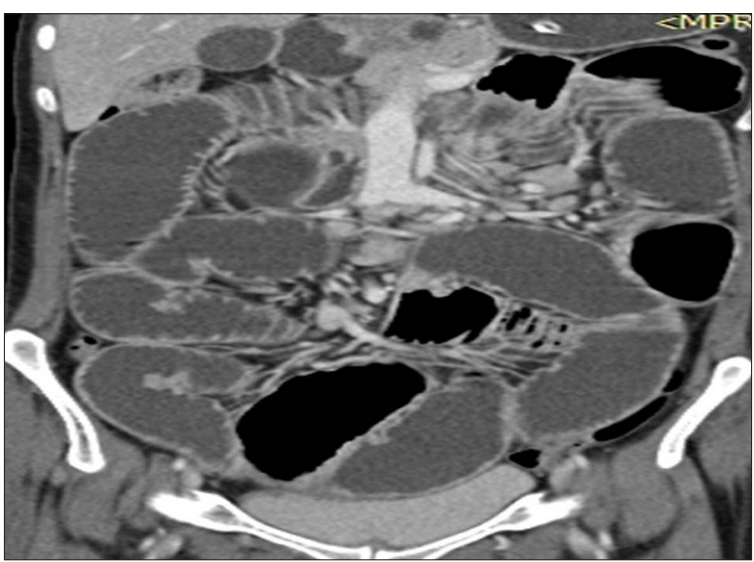

Figure 1. MDCT enterography, coronal MPR images showing welldistended jejunum and ileal loops with normal bowel wall enhancement.

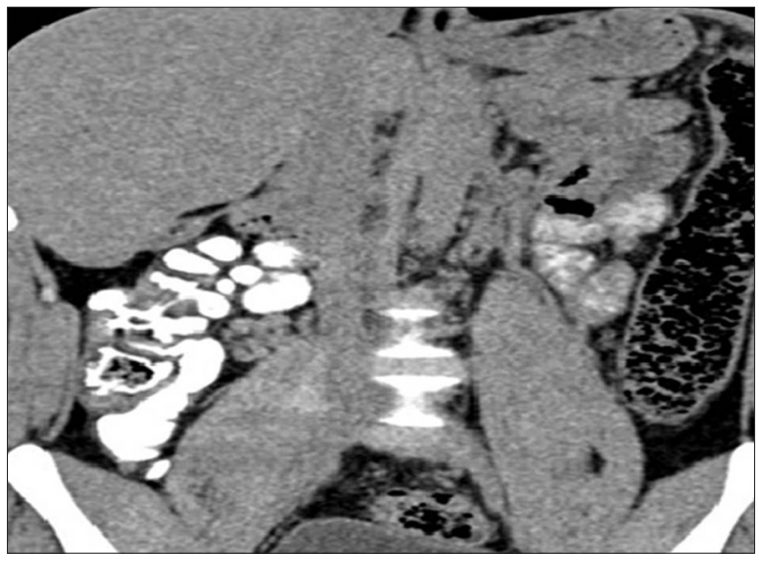

Figure 2. MDCT enterography, coronal MPR image with positive bowel contrast showing a normal IC junction.

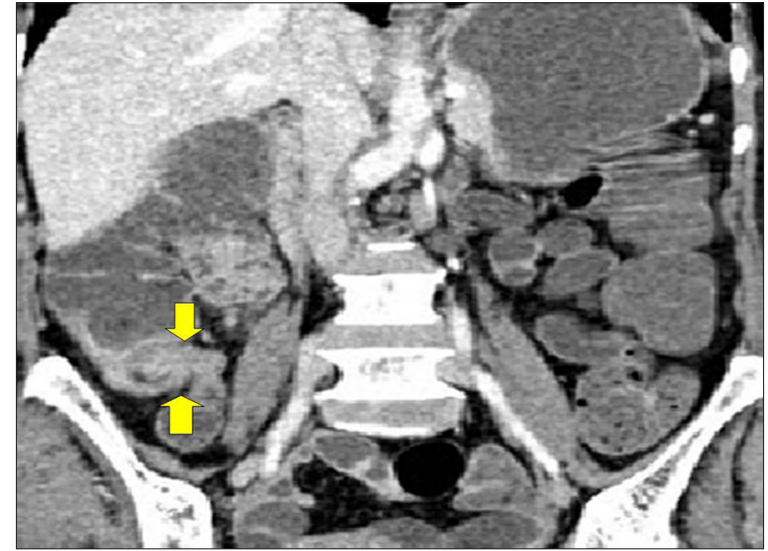

Figure 3. MDCT enterography, coronal MPR images showing a circumferential, enhancing mural thickening involving the ileocaecal junction without stricture (arrows). Also noted is an enhancing caecal wall thickening of the lateral wall.

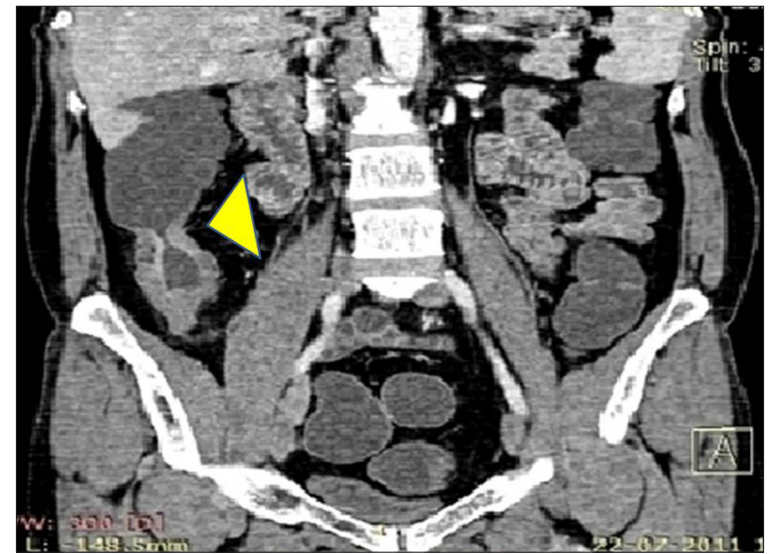

Figure 4. MDCT enterography, coronal MPR images showing a concentric, enhancing mural thickening of the ileocaecal junction and the terminal ileum with an altered ileocaecal angle (arrow-head).

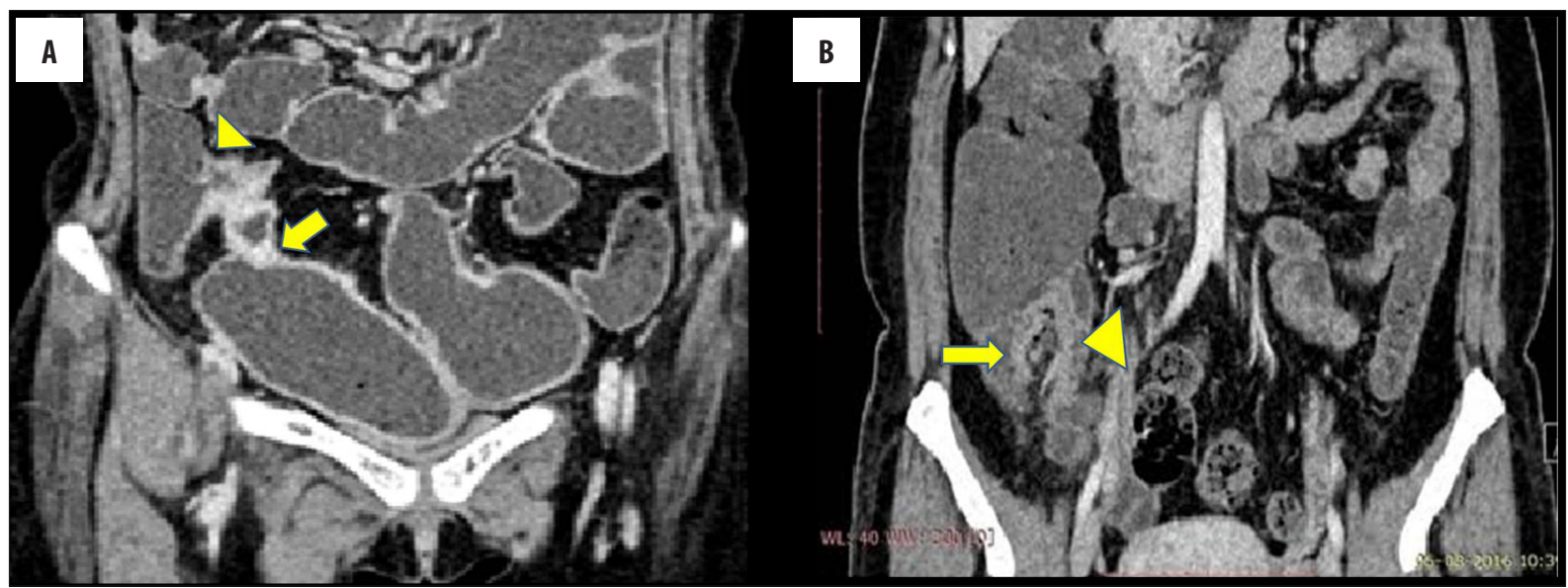

Figure 5. (A) MDCT enterography, coronal MPR images demonstrating a concentric, enhancing mural thickening of the ileocaecal junction (arrowhead) and concentric stricture of the terminal ileum (arrow) causing dilatation of the proximal ileal loop. (B) MDCT enterography, coronal MPR images demonstrating an asymmetric wall thickening of the ileocaecal junction (arrow-head) involving the medial wall of the caecum (arrow) and terminal ileum. Pericaecal fat stranding is also evident in the image 


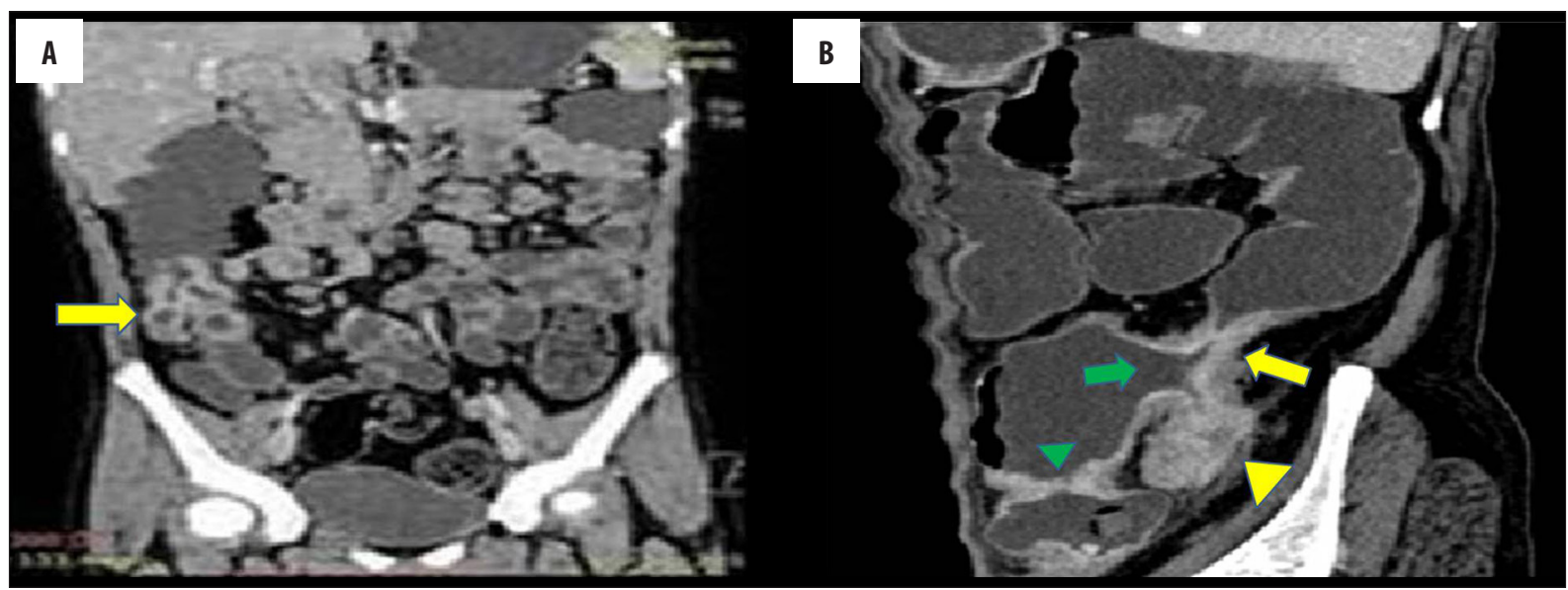

Figure 6. (A) MDCT enterography, coronal MPR image demonstrating a contracted, pulled-up caecum (arrow). Also seen are multiple mesenteric nodes. (B) MDCT enterography, sagittal MPR images demonstrating a contracted caecum with completely obliterated lumen (arrow head) in the normal position with involvement of the contiguous ascending colon (arrow). Note is made of a patulous ileocaecal junction opening directly into the ascending colon (green arrow). A napkin ring stricture in the terminal ileum (green arrow head) is also evident.

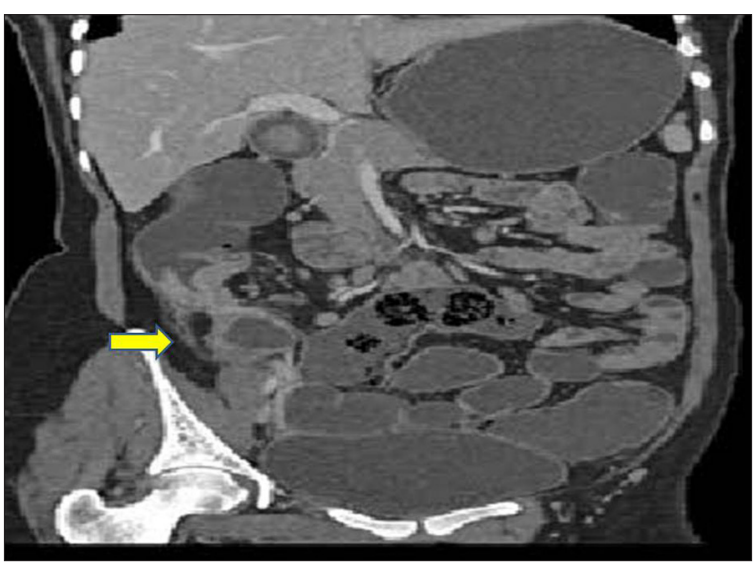

Figure 7. MDCT enterography, oblique coronal image showing a high-placed, contracted caecum (arrow) with complete obliteration of its lumen, symmetrical thickening of the ascending colon and the IC junction. Few sub-centimetre mesenteric lymph nodes are also seen.

mild-to-moderate enhancement, may be encountered with involvement of the IC valve. The thickening is usually symmetrical (Figure 5A) or sometimes asymmetrical and limited to the medial wall (Figure 5B).

The caecum may be contracted, demonstrate a small calibre, and appear pulled-up (Figure 6A) with contiguous involvement of the ascending colon (Figure 6B)

An abnormally high placed, contracted, distorted caecum without any mural thickening suggests the presence of fibrosis [13], which is specific for tuberculosis (Figure 7).

A patulous IC valve and loss of the IC angle is also known [14-16], which lead to a patulous IC junction opening directly into the ascending colon (Figure 6B) with a completely obliterated caecal lumen.

The ileum is far more commonly involved than the jejunum due to the abundance of lymphoid tissue (Peyer patches) in the distal and terminal ileum [13]. The terminal part of the

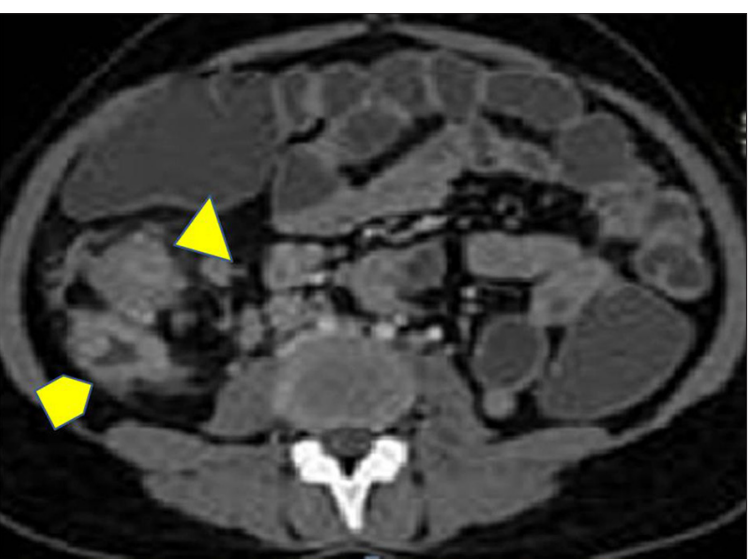

Figure 8. MDCT enterography, axial image demonstrating the hypertrophic type of ileocaecal tuberculosis with a thickened and contracted caecum (arrow) and gross thickening and luminal obliteration of the ileocaecal junction (arrowhead). Also noted are small perienteric enlarged lymph nodes with necrotic fat. Perienteric stranding and focal peritoneal thickening is also seen.

ileum, ileocaecal (IC) junction, and the caecum are concomitantly involved in the majority of patients. Mural thickening of the caecum or ileum with s simultaneous involvement of the IC junction is strongly suggestive of tuberculosis [12].

\section{Nature of Bowel Involvement}

\section{Bowel wall thickening}

Focal, multifocal, or segmental involvement is seen as a concentric symmetrical/asymmetrical mural thickening showing a mild-to-moderate homogenous enhancement with or without a resultant luminal narrowing (Figure 8). The typical concentric mural thickening is the commonest CT finding. (Figures 9, 10). The wall thickening is homogenous and no stratification is evident like in Crohn's disease [17]. 


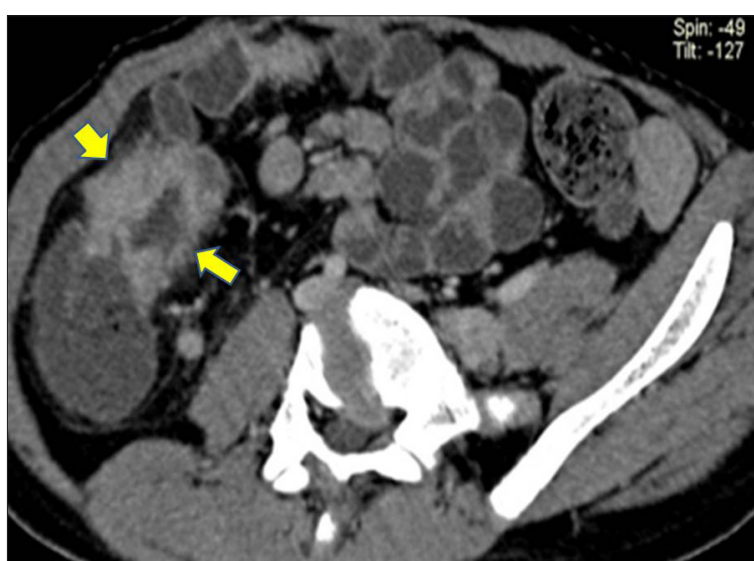

Figure 9. MDCT enterography, oblique axial MPR image showing an asymmetric thickening of the ileocaecal region and terminal ileum (arrows) with perienteric stranding and surrounding enlarged lymph nodes.

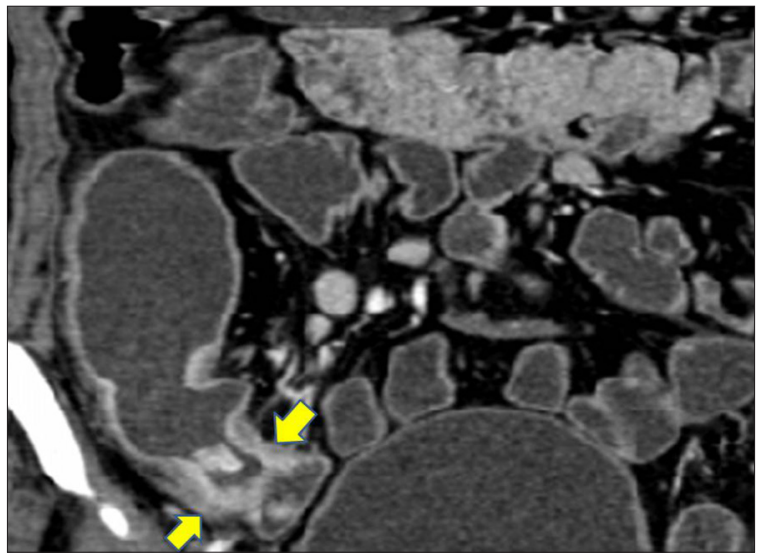

Figure 10. MDCT enterography, coronal MPR image demonstrating a concentric, enhancing mural thickening (arrows) of the ileal wall. Dilatation of the bowel loop and presence of an enterolith suggest small bowel obstruction. Also noted are enhancing, enlarged mesenteric lymph nodes.

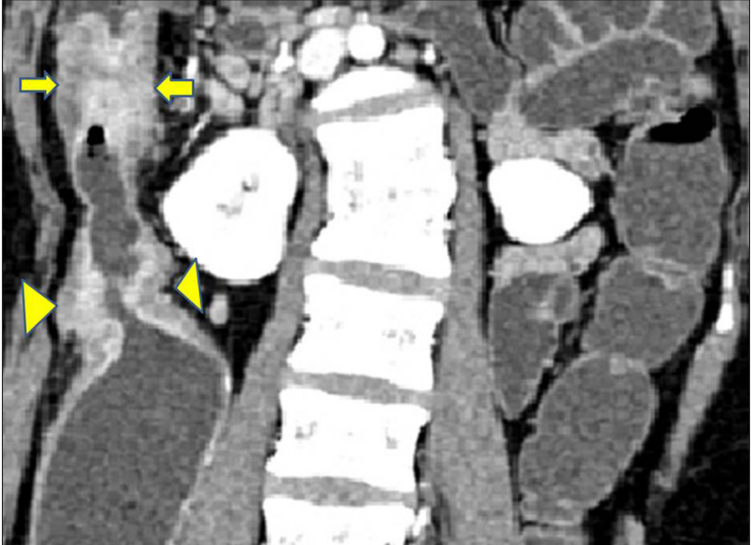

Figure 11. MDCT enterography, coronal MPR images showing an enhancing bowel wall thickening causing luminal compromise affecting the IC junction (arrow-heads) and ascending colon with near-total luminal obliteration in the later (arrows). Also noted is scoliosis of the lumbar spine with obliteration of disc space between L1-L2 vertebrae, suggestive of TB spondylitis.

Multifocal thickening is more common than focal thickening of the bowel (Figure 11), and concurrent IC involvement is a diagnostic clue. Enhancement of the bowel wall in the region of focal or segmental mural thickening is present and is mild-to-moderate and homogenous in nature.

Perienteric stranding without increased vascularity is also seen in association with bowel wall thickening, reflecting the inflammatory nature of the disease (Figure 9).

\section{Abnormal calibre}

Jejunal and ileal calibre more than 3.0 and $2.5 \mathrm{~cm}$ respectively may be evident, usually with the presence of a narrowed bowel segment beyond the dilatation. Less commonly, a segmental/diffuse dilatation may be seen in cases presenting with malabsorption due to GI TB. Sometimes, peritoneal TB can cause small bowel involvement leading to small bowel dilation.

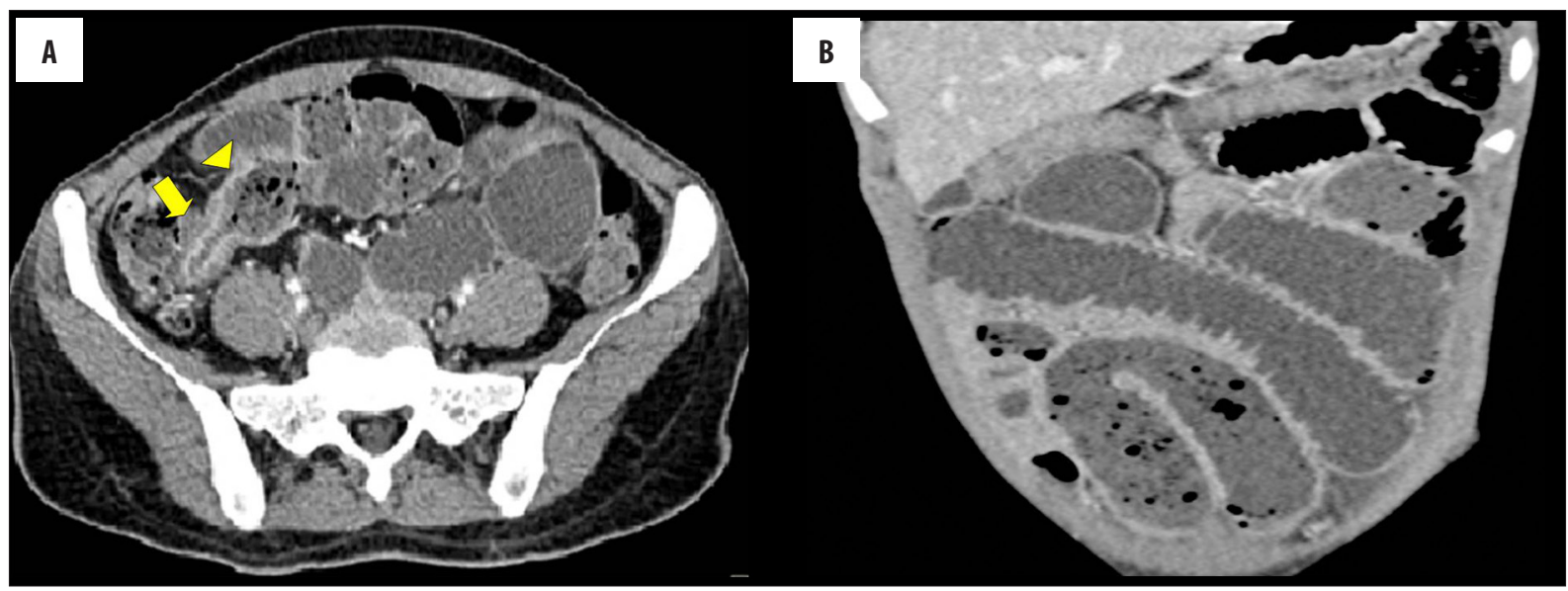

Figure 12. (A) MDCT enterography, axial image showing a stricture at the ileocaecal junction (arrow) leading to dilation of the terminal ileal loop, demonstrating the small bowel faeces sign (arrowhead). (B) MDCT enterography, coronal MPR images showing dilated jejunal and ileal loops with the small bowel faeces sign in ileal loops. 


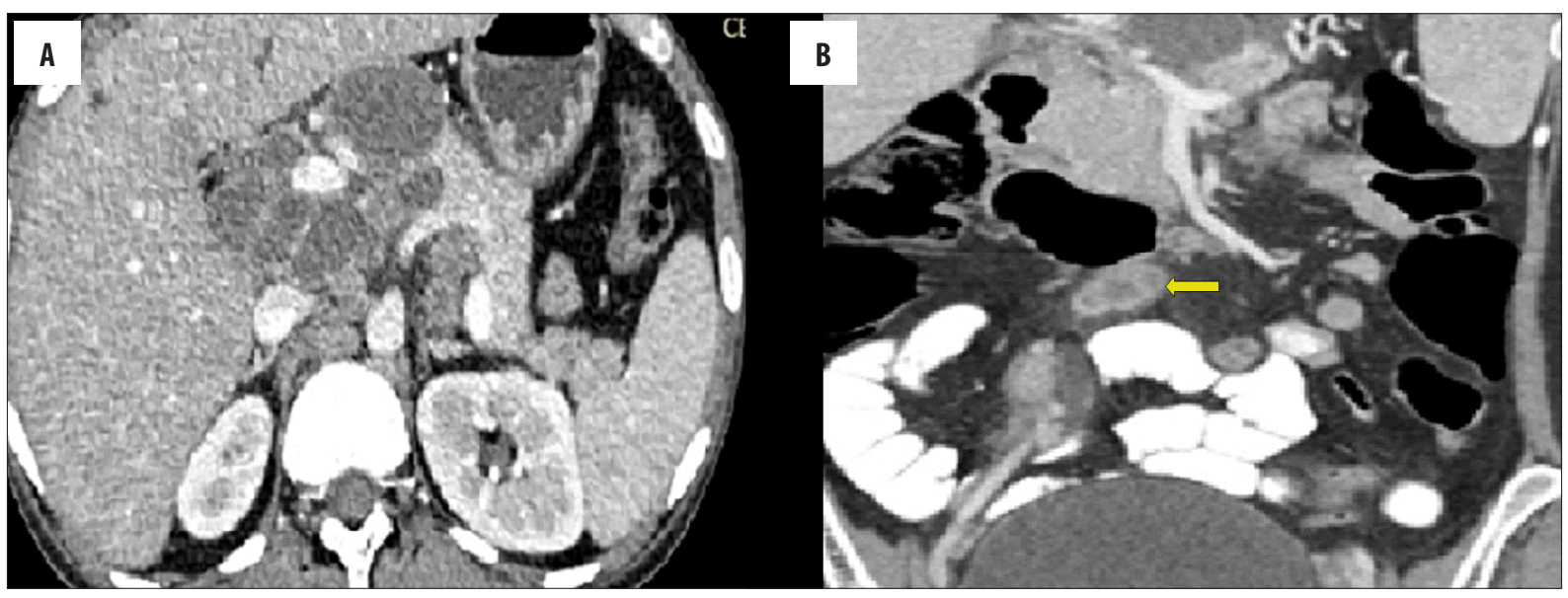

Figure 13. (A) MDCT enterography, axial image showing necrotic, periportal, peripancreatic, pancreaticoduodenal, and retroperitoneal lymphadenopathy. (B) MDCT enterography, coronal MPR image demonstrating multiple enlarged necrotic mesenteric lymph nodes (arrow). Central necrosis with peripheral contrast enhancement suggests caseation which is diagnostic for tuberculosis.

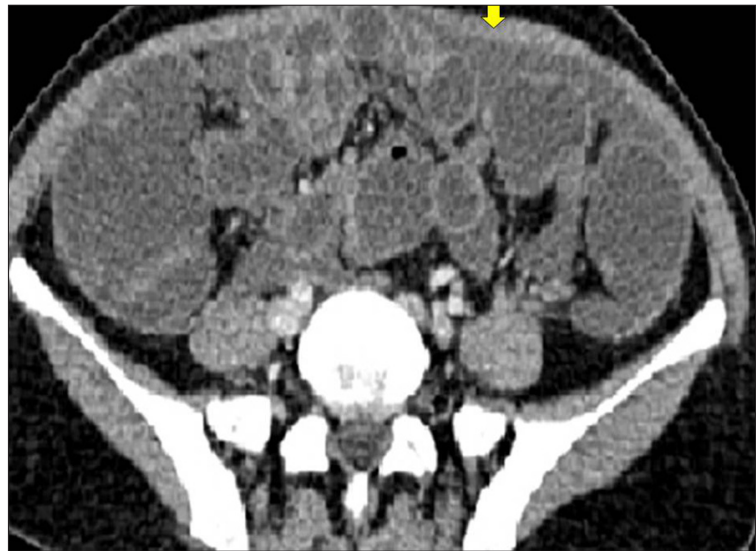

Figure 14. MDCT enterography, axial image showing interbowel fluid collections (arrow) with segmental bowel dilatation.

Identification of a transition zone with proximal dilation and distal normal or collapsed bowel loops suggests stricture. An inflammatory stricture appears as a circumferential, enhancing wall thickening that causes luminal compromise (Figure 12A) leading to proximal small bowel dilatation and other ancillary signs like the small bowel faeces sign (Figure 12B) and enteroliths.

\section{Active bowel disease}

An enhancing, circumferential bowel wall thickening in the terminal ileum, ileocaecal junction, and the caecum suggest tubercular disease, which may or may not be associated with luminal obstruction. Active disease is associated with wall thickening with enhancement of the entire wall or only of the mucosa [17]. Lack of enhancement of thickened wall segments, that cause proximal bowel dilatation, suggests a chronic healed stricture [17]. Peri-enteric changes of fat stranding with increased vascularity may be evident in association with disease activity. Strong supportive evidence is provided by enlarged enhancing mesenteric lymph nodes with central necrosis.

An objective, CT-based predictive model has been suggested to diagnose GI TB using three CT parameters - long

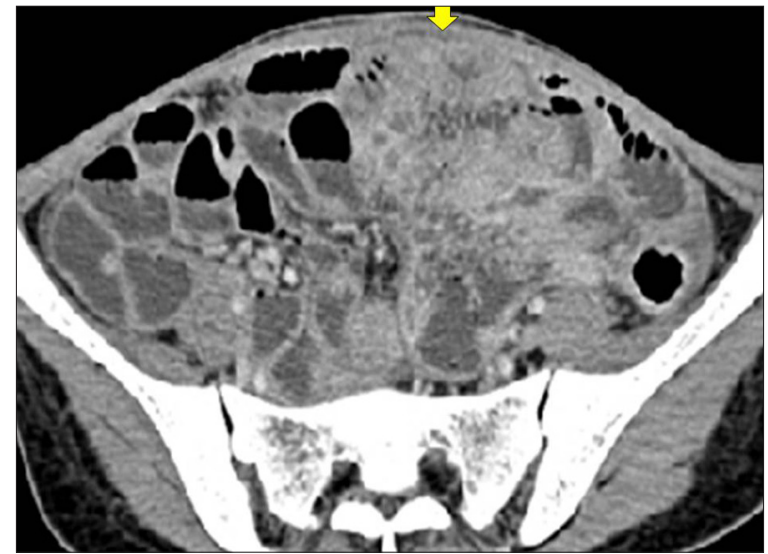

Figure 15. MDCT enterography, axial images demonstrating matting of jejunoileal loops (down arrows) with segmental dilatation and multiple air-fluid levels.

segment bowel wall involvement, terminal ileum involvement with or without cecum affection, and abdominal lymph node size greater than 1 centimetre [18].

\section{Extra intestinal findings}

Sometimes, bowel involvement occurs due to the presence of extraintestinal disease.

\section{Lymph nodes}

Lymphadenopathy (short axis diameter more than $1 \mathrm{~cm}$ ) is the commonest extraintestinal finding that is seen in almost two-thirds of patients with GI tuberculosis and in $55-66 \%$ of patients with abdominal tuberculosis[18]. Involvement of multiple lymph node groups is seen most commonly with mesenteric lymphadenopathy (Figure 13A).

Periportal lymphadenopathy also suggests tuberculosis, and $40-60 \%$ of patients with lymphadenitis classically demonstrate a hypoattenuating centre and an enhancing rim suggestive of caseous necrosis [19]. (Figure 13A, 13B). Sometimes, the enlarged lymph nodal masses may cause extrinsic bowel compression. 


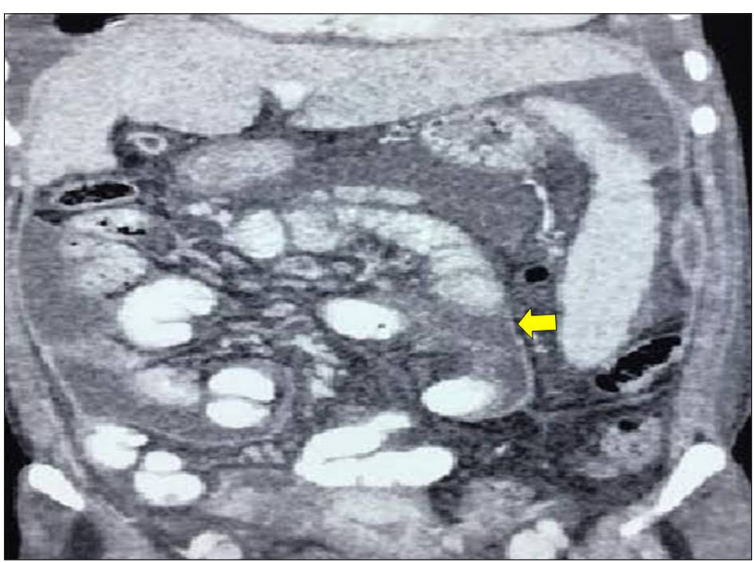

Figure 16. MDCT enterography, coronal image showing a thin membrane (arrow) around the matted jejunoileal loops, indicating formation of a cocoon. The loops inside the cocoon appear prominent.

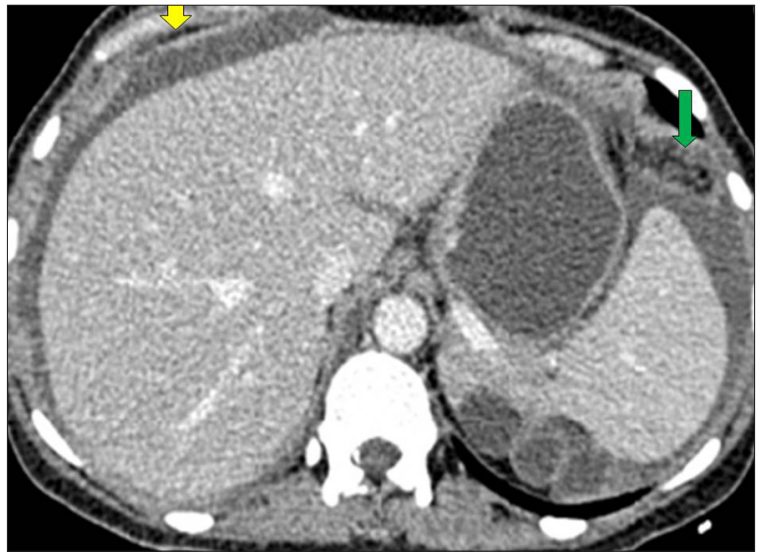

Figure 17. MDCT enterography, axial image showing omental nodules (green arrow) with enhancing thickened peritoneum. Also noted in the right hypochondrium is peritoneal thickening with ascites (yellow arrow).

\section{Peritoneum}

Tubercular peritonitis, that is found in three types - wet, fibrotic, and dry, has overlapping CT features [19] with segmental enhancing plaques like nodular peritoneal thickening, or loculated hyperdense collections and ascites.

Wet tubercular peritonitis causes the bowel loops to adhere together due to thick exudates. Matting of bowel loops with or without evidence of segmental dilation is a significant finding

pointing towards the diagnosis of tuberculosis. Interbowel collections may also be seen (Figure 14).

Small loculated collections are better appreciated with the use of positive oral contrast to distend the bowel. Matting may involve jejunal loops as well but is more commonly found in the ileal region. (Figure 15).

Sometimes, a membrane forms around the matted small bowel loops, forming a cocoon, entrapping the bowel loops, and causing their obstruction [21] (Figure 16).

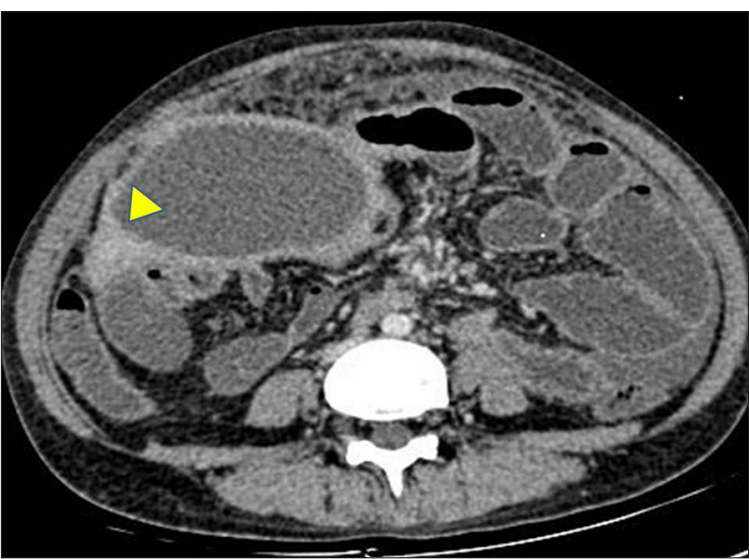

Figure 18. MDCT enterography, axial image showing a walledoff mesenteric collection (arrow head) displacing and compressing the adjacent ileal loops in the right iliac fossa. The proximal small bowel has an increased luminal diameter and air-fluid levels suggestive of low-grade obstruction. The omentum also appears thickened and stranded.

\section{Mesentery and Omentum}

Omental thickening and nodularity is seen with its involvement [21] (Figure 17).

Mesenteric collections/abscesses may occur due to tubercular adenitis and can cause extrinsic bowel compression with or without significant bowel obstruction (Figure 18).

Rarely, enteric fistulas can be seen in GI tuberculosis with extensive peritoneal involvement, and they are demonstrated better with positive oral contrast. Fistulas occur more commonly with the anterior abdominal wall than with the bowel and rarely with other hollow organs (Figures 19A-19C). Sometimes, CT sinography/fistulography using positive water soluble contrast has to be undertaken to demonstrate the complete extent of the fistula.

\section{Associated Findings}

Solid organs- hepatosplenomegaly with or without multiple hypodense lesions, suggestive of granulomas, may be seen (Figure 20).

\section{Miscellaneous Findings}

Psoas abscesses (Figure 21), pleural effusion (Figures 20, 22 ), and complex adnexal lesions could be associated and can be well demonstrated on MDCTE in GI TB.

\section{Post-Treatment Findings}

Post-treatment findings of $\mathrm{I} / \mathrm{C}$ tuberculosis vary from none to contracted and high-placed caecum with or without a stricture or distortion of the IC valve (Figure 23).

A fibrotic stricture, formed post treatment, demonstrates a marked luminal narrowing with a remarkable transition zone and no associated wall thickening (Figure 24). 


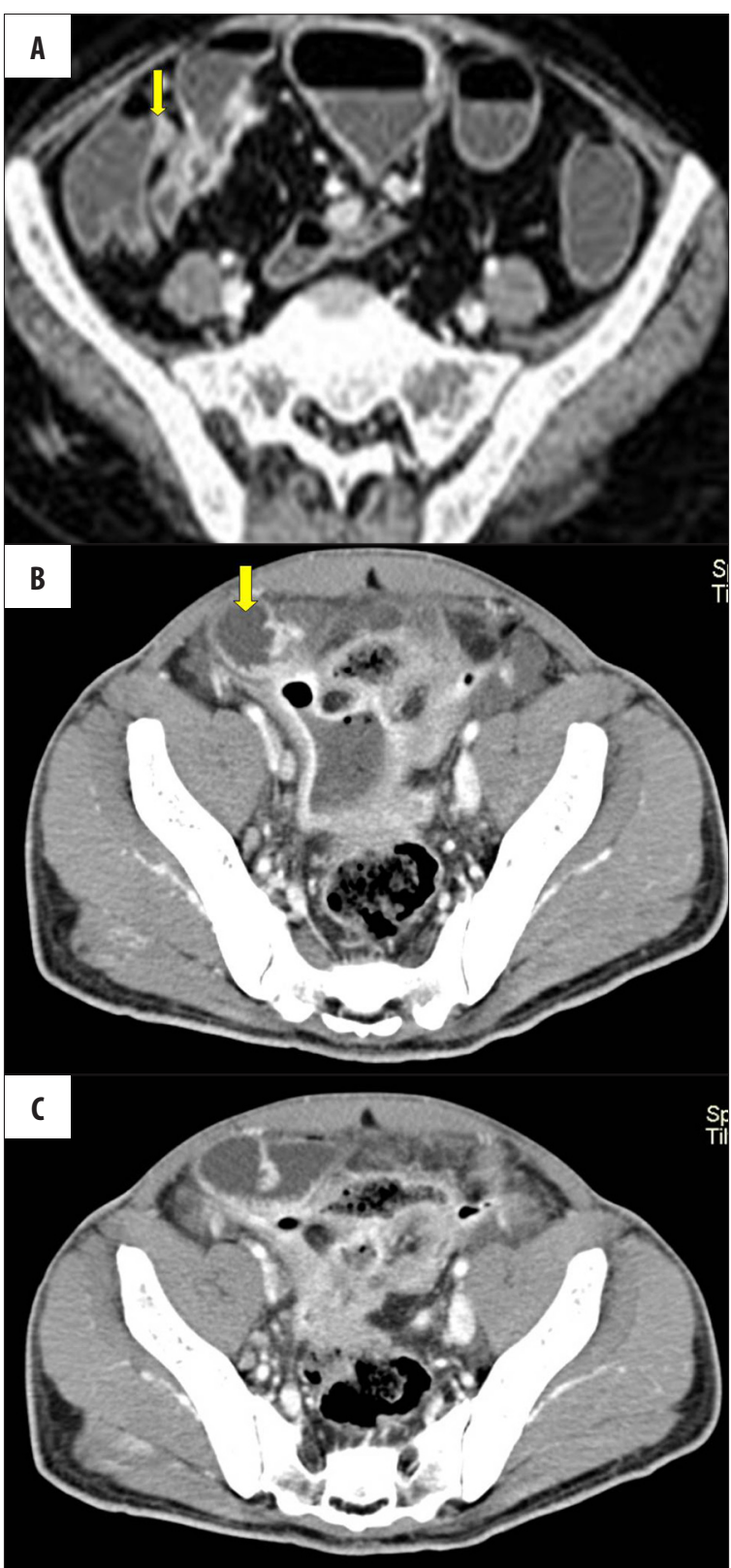

Figure 19. (A) Axial MDCT enterography images demonstrating a mild obstructive thickening and narrowing of the terminal ileum with an enhancing tract (arrow) communicating with the colon, suggestive of an iliocolic fistula. (B, C) MDCT enterography, axial images demonstrating an enhancing, walled-off collection with air foci in the pelvis which extends laterally up to the ileal loop (arrow). On surgery, this collection was found to be directly communicating with the ileal loop, suggesting an ileal perforation with formation of a fistula between the ileum and peritoneal cavity.

Concurrent findings of lymph-nodal, peritoneal, or omental calcifications may be found.

\section{Conclusions}

Adequate luminal distention and the use of negative contrast to assesses the bowel wall, in addition to the

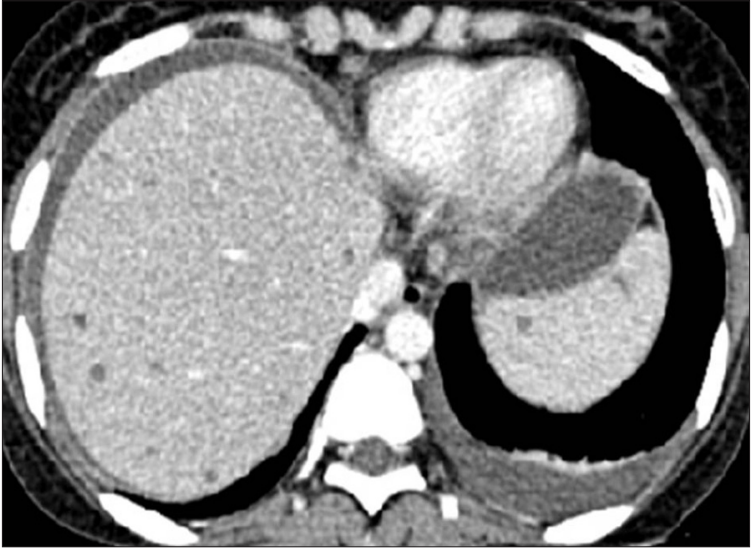

Figure 20. Axial MPR contrast enhanced images demonstrating multiple hypodense spleen and liver lesions suggestive of granulomas. Also noted is left pleural effusion and ascites.

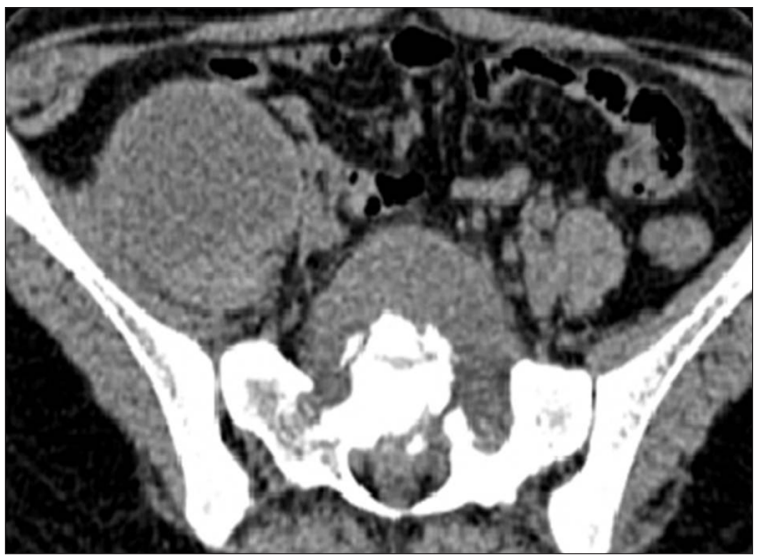

Figure 21. Axial scans demonstrating an abscess in the iliopsoas on the right side. A presacral abscess with vertebral body destruction is also evident.

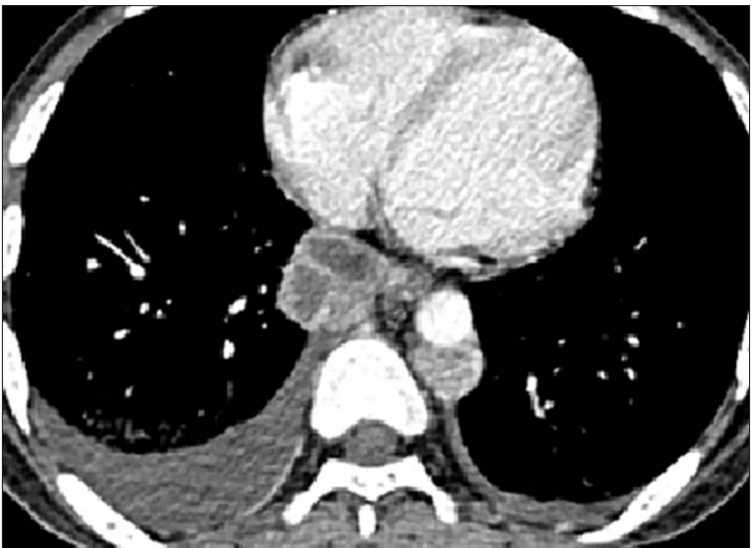

Figure 22. Axial scans demonstrating right pleural effusion and bilateral pleural thickening with necrotic posterior mediastinal lymphadenopathy.

perienteric findings and the associated solid organ, peritoneal, and mesenteric involvement, makes MDCT enterography a one-stop imaging modality for diagnosing GI involvement in tuberculosis. 


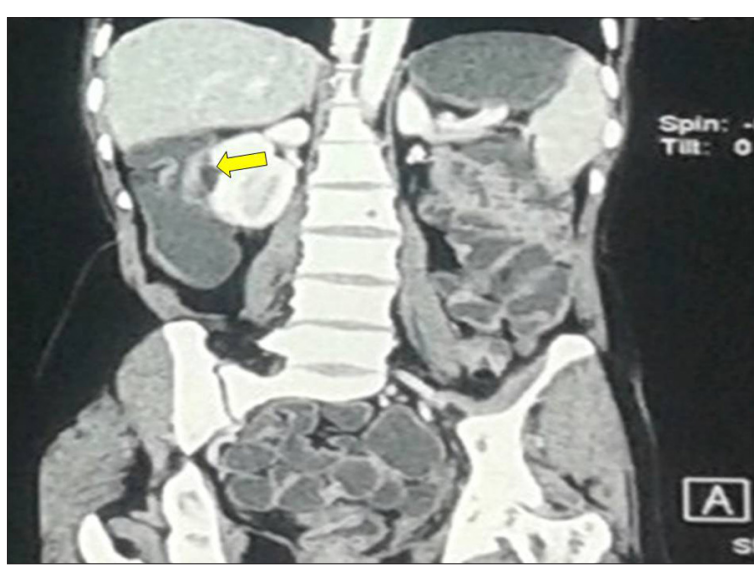

Figure 23. Post-treatment ileocaecal TB: MDCT enterography, coronal MPR image showing a high-placed sub-hepatic caecum with a fibrotic partial stricture of the IC junction (arrow).

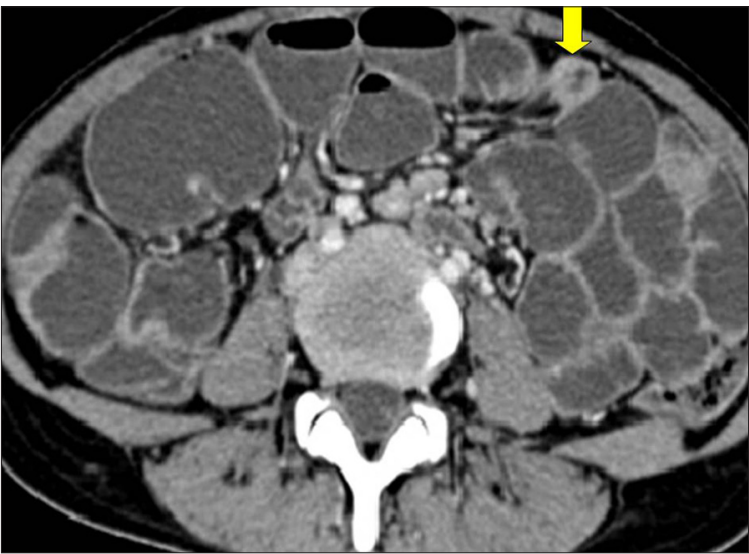

Figure 24. MDCT enterography, axial image shows a remarkable transition zone with relative narrowing of the bowel lumen without any wall thickening (arrow) with evidence of dilated bowel loops suggestive of a fibrotic stricture after completion of ATT.

\section{References:}

1. Kalra N, Agrawal P, Mittal V et al: Spectrum of imaging findings on MDCT enterography in patients with small bowel tuberculosis. Clin Radiol, 2014; 69(3): 315-22

2. Tandon RK, Sarin SK, Bose SL et al: A clinico-radiological reappraisal of intestinal tuberculosis - changing profile? Gastroenterol Jpn, 1986; 21: 17-22

3. Raptopoulos V, Schwartz RK, McNicholas MM et al: Multiplanar helical CT enterography in patients with Crohn's disease. Am J Roentgenol, 1997; 169: 1545-50

4. Paulsen SR, Huprich JE, Fletcher JG et al: CT enterography as a diagnostic tool in evaluating small bowel disorders: Review of clinical experience with over 700 cases. Radiographics, 2006; 26: 641-62

5. Ilangovan R, Burling D, George A et al: CT enterography: Review of technique and practical tips. Br J Radiol, 2012; 85(1015): 876-86

6. Hara AK, Leighton JA, Sharma VK et al: Imaging of small bowel disease: Comparison of capsule endoscopy, standard endoscopy, barium examination, and CT. Radiographics, 2005; 25(3) 697-711

7. Macari M, Megibow A, Balthazar E: A pattern approach to the abnormal small bowel: Observations at MDCT and CT enterography. Am J Roentgenol, 2007; 188: 1344-55

8. Siddiki H, Fletcher JG, Hara AK et al: Validation of a lower radiation computed tomography enterography imaging protocol to detect Crohn's disease in the small bowel. Inflamm Bowel Dis, 2011; 17: $778-86$

9. DelGaizo AJ, Fletcher JG, Yu L et al: Reducing radiation dose in CT enterography. Radiographics, 2013; 33: 1109-24

10. Chong VH, Lim KS: Gastrointestinal tuberculosis. Singapore Med J, 2009; 50: 638-45
11. Kapoor VK: Abdominal tuberculosis. Postgrad Med J, 1998; 74: 459-67

12. Suri S, Gupta S, Suri R: Computed tomography in abdominal tuberculosis. Br J Radiol, 1999; 72: 92-98

13. Tripathi PB, Amarapurkar AD: Morphological spectrum of gastrointestinal tuberculosis. Trop Gastroenterology, 2009; 30(1): 35-39

14. Hussain T, Salamat A, Farooq MA et al: Indications for endoscopic ultrasound and diagnosis on fine-needle aspiration and cytology.J Coll Physicians Surg Pak Apr, 2009; 19(4): 223-27

15. Ozbülbül NI, Ozdemir M, Turhan N: CT findings in fatal primary intestinal tuberculosis in a liver transplant recipient. Diagn Interv Radiol, 2008; 14(4): 221-24

16. Barreiros AP, Braden B, Schieferstein-Knauer C et al: Characteristics of intestinal tuberculosis in ultrasonographic techniques. Scand J Gastroenterol, 2008; 43(10): 1224-31

17. Kalra N, Agrawal P, Mittal V et al: Spectrum of imaging findings on MDCT enterography in patients with small bowel tuberculosis. Clin Radiol, 2014; 69: 315-22

18. Kedia S, Sharma R, Nagi B et al: Computerized tomography-based predictive model for differentiation of Crohn's disease from intestinal tuberculosis. Indian J Gastroenterol, 2015; 34: 135-43

19. Zissin R, Gayer G, Chowers M et al: Computerized tomography findings of abdominal tuberculosis: Report of 19 cases. Isr Med Assoc J, 2001; 3(6): 414-18

20. Leder RA, Low VH: Tuberculosis of the abdomen. Radiol Clin North Am, 1995; 33: 691-705

21. Sharma R, Madhusudhan KS, Ahuja V: Intestinal tuberculosis versus crohn's disease: Clinical and radiological recommendations. Indian J Radiol Imaging, 2016; 26(2): 161-72 